mechanism of homoeostasis in developing plant tissues. Some of the results obtained on the effect of indol-3-ylacetic acid on enzyme induction in germinating seeds of Bengal gram (Cicer arietinum) are presented.

Previous findings on the degradation of phosphatidylcholine during germination of this legume with the concomitant appearance of a membranebound phospholipase D (Talwalkar et al., 1964, 1969) have been supplemented by the demonstration of degradation of protein and nucleic acid (Hadi, 1966) as well as the activation of ribosomal function during germination (Hadi \& Krishna Murti, 1968).

To explore whether amino acids arising from protein degradation, apart from enriching the amino acid pool, were being channelled into alternative pathways and utilized for the synthesis of physiologically active non-protein nitrogenous compounds, the activities of enzymes known to mediate the metabolism of amino acids were investigated in gram seedlings. Tryptophan oxygenase, glutamate dehydrogenase, tyrosine transaminase and indol-3ylacetate oxidase were not detectable in the dormant seeds but made their appearance after $48 \mathrm{~h}$ germination. During this period there was 1000 -fold stimulation of peroxidase activity compared with that of the dormant seed as well as significant increases in the pools of soluble carbohydrates and amino acids. Cycloheximide or azauridine, when present in the aqueous medium used for germination, not only inhibited elongation of the seedlings but also suppressed the induction of peroxidase and the accumulation of free carbohydrates and amino acids.

When induction was studied with seedlings in vitro incubated with substrate it was found that indol-3ylacetic acid $(1 \mu \mathrm{M})$ or cyclic AMP (adenosine $3^{\prime}: 5^{\prime}$-cyclic monophosphate) (0.1 mM) added exogenously to the medium stimulated tryptophan oxygenase activity two- to three-fold in 48-96h seedlings but did not affect the activity of tyrosine aminotransferase or glutamate dehydrogenase. The stimulation of tryptophan oxygenase activity by indol-3ylacetic acid in vitro was inhibited by actinomycin D and cycloheximide. The stimulation of tryptophan oxygenase activity by indol-3-ylacetic acid or cyclic AMP was accompanied by increased incorporation of $\mathrm{DL}-\left[1-{ }^{14} \mathrm{C}\right]$ valine into proteins. Indol-3-ylacetic acid also stimulated the incorporation of $\left[8-{ }^{14} \mathrm{C}\right]-$ adenine into cyclic AMP in 48-72h seedlings, from which it was inferred that indol-3-ylacetic acid presumably triggered a mechanism mediating the synthesis of cyclic AMP (Azhar \& Krishna Murti, $1971 a, b)$.

The relative rates of synthesis of cyclic AMP, indol-3-ylacetic acid, peroxidase and tryptophan oxygenase have been followed during germination to establish the correlation, if any, between enzyme synthesis and the appearance of indol-3-ylacetic acid or its mediator cyclic AMP. Purification of tryptophan oxygenase from induced and non-induced seedlings has been carried out to enable investigations on the modulation of its activity by indol-3-ylacetic acid.

Azhar, S. \& Krishna Murti, C. R. (1971a) Biochem. Biophys. Res. Commun. 43, 53

Azhar, S. \& Krishna Murti, C. R. (1971b) Indian J. Biochem. 8, in the press

Hadi, S. M. (1966) Indian J. Biochem. 3, 203

Hadi, S. M. \& Krishna Murti, C. R. (1967) Indian J. Biochem. 4, 10

Talwalkar, R. T., Garg, N. K. \& Krishna Murti, C. R. (1964) Indian J. Exp. Biol. 2, 37

Talwalkar, R. T., Garg, N. K. \& Krishna Murti, C. R. (1969) Indian J. Biochem. 6, 229

\section{Regulation of Phosphoglucomutase}

By B. D. Sanwal, H. W. Duckworth and M. L. Hollier (Department of Medical Cell Biology, Medical Sciences Building, University of Toronto, Toronto 181, Ont., Canada)

Phosphoglucomutase from at least two sources, Escherichia coli and rabbit muscle, is strongly and specifically inhibited by the high-energy metabolites GTP and acetyl-CoA. GTP acts as its magnesium complex. Detailed kinetic investigations reveal that the two inhibitors are non-competitive with glucose 1-phosphate, glucose 1,6-diphosphate and $\mathrm{Mg}^{2+}$, and that they bind to the enzyme at separate sites. The inhibitors act in a hyperbolic manner, and do not induce sigmoid kinetics in the saturation curves for the substrates.

All previous workers with phosphoglucomutase have agreed that the enzyme consists of one polypeptide chain of molecular weight 62000 . This enzyme therefore seems to be a unique example of an allosteric enzyme without subunits. We have reinvestigated the subunit structure to check this possibility. When rabbit muscle phosphoglucomutase, which gives a single band on polyacrylamide gels, is submitted to the sodium dodecyl sulphate-polyacrylamide electrophoresis technique, about $20-30 \%$ of the material migrates at a rate corresponding to a molecular weight of $68000-74000$, but the remaining $70-80 \%$ forms a clear band at a position corresponding to a molecular weight of 31000 . This behaviour is shown by both the phospho- and the dephosphoenzyme. Results obtained by a short-column sedimentation-equilibrium technique show that in buffer at $\mathrm{pH} 7.0$, both in the presence and in the absence of guanidine hydrochloride, the molecular weight of phosphoglucomutase is 62000. Reduced and carboxymethylated phosphoglucomutase, however, shows considerable heterogeneity (molecular weights 
in the range $30000-120000$ ). In $0.1 \%$ sodium dodecyl sulphate-2-mercaptoethanol the enzyme behaves as a homogeneous species whose molecular weight, after correction for a large amount of sodium dodecyl sulphate binding, is $\mathbf{3 1 0 0 0}$.

By the use of dansylation procedures equal amounts of $N$-terminal lysine and valine are detected for both the phospho- and the dephospho-enzyme. The latter, when subjected to the carbamoylation technique of Stark \& Smythe (1963), gives 0.90 $1.09 \mathrm{~mol}$ of valine $/ 62000 \mathrm{~g}$ of phosphoglucomutase. The evidence indicates that phosphoglucomutase is a dimer of subunits that are about the same size but have different $N$-terminal residues.

Stark, G. R. \& Smythe, D. G. (1963) J. Biol. Chem. 238, 214

\section{Studies on Tissue Cultures of Maize, Wheat, Rice and Sorghum}

By R. R. Hendre, A. F. Mascarenhas, Meera Pathak, B. Seetarama Rao and V. Jagannathan (National Chemical Laboratory, Poona-8, India)

Tissues of monocotyledonous plants have been more difficult to grow in vitro than dicotyledonous tissues. Callus cultures of maize (Zea mays), wheat (Triticum vulgare), rice (Oryza sativa) and sorghum (Sorghum vulgare) were obtained and have been shown to be viable on prolonged subculture in vitro for 3-6 years (Mascarenhas et al., 1969). This is the first report of viable callus and root cultures of maize and sorghum. The nutrition and differentiation of these tissues were studied. Good growth was obtained on chemically defined media consisting of inorganic salts, sucrose, an amino acid mixture, vitamins and auxin. No requirement for a cytokinin, gibberellin or coconut milk could be demonstrated. Naphth-1ylacetic acid (5-10p.p.m.) gave maximum growth. The growth curves of maize and wheat in liquid culture and the utilization of sucrose, N, P and Fe were determined.

Maize, wheat and sorghum showed the unusual property of growing mostly as callus on agar, whereas on the same medium in shake flasks without agar they grew only as a mass of roots. The roots grew again as callus when transferred back to agar. This differentiation and de-differentiation of maize, wheat and sorghum tissues are unique, since they require no change in the composition of the medium and are readily reversible.

Mascarenhas, A. F., Hendre, R. R., Seetarama Rao, B. \& Jagannathan, V.(1969) Indian J.Exp. Biol.7,65
Role of 8-Aminolaevulinate Dehydratase in the Regulation of Haem Synthesis

By G. Padmanaban and K. Malathi (Department of Biochemistry, Indian Institute of Science, Bangalore-12, India)

It is believed that $\delta$-aminolaevulinate dehydratase is not a rate-limiting enzyme in the pathway of haem biosynthesis, since it has been found to be present in considerable excess of $\delta$-aminolaevulinate synthetase, the first enzyme of the pathway, in animal and bacterial systems (Lascelles, 1964). However, detailed investigations with Neurospora crassa have shown that the enzyme is rate-limiting in this organism, and that it can be induced by iron and repressed by protoporphyrin (Muthu Krishnan et al., 1968, 1969). Neurospora extracts contain a protein inhibitor for the enzyme and an unidentified low-molecular-weight activator. The enzyme is also subject to feedback inhibition by coproporphyrinogen III. The existence of so many controls on the enzyme indicates its regulatory nature, which is also supported by the rapid turnover of this protein in the organism, the half-life for the induced enzyme being $4.5 \mathrm{~min}$. The possible regulation of this enzyme at the translational level and transcriptional level will be discussed. $\delta$-Aminolaevulinate dehydratase activity has also been found to be low in normal yeast, where it appears to have a role in haem synthesis and mitochondrogenesis (Jayaraman et al., 1971). However, the enzyme is present at very high activities in photosynthetic bacteria, higher plants and animal systems. Yeast and fungi appear to constitute a separate class where the enzyme is ratelimiting, and the protozoan Euglena gracilis exhibits an intermediate behaviour.

Jayaraman, J., Padmanaban, G., Malathi, K. \& Sarma, P. S. (1971) Biochem. J. 121, 531

Lascelles, J. (1964) Tetrapyrrole Biosynthesis and its Regulation, W. A. Benjamin, New York

Muthu Krishnan, S., Padmanaban, G. \& Sarma, P. S. (1968) Biochem. Biophys. Res. Commun. 31, 333

Muthu Krishnan, S., Padmanaban, G. \& Sarma, P. S. (1969) J. Biol. Chem. 244, 4241

\section{Regulation of Coenzyme Nucleotide Metabolism in Plants}

By S. D. Ravindranath and N. Appaji Rao (Department of Biochemistry, Indian Institute of Science, Bangalore-12, India)

The concentrations of coenzyme nucleotides could be controlled by environmental conditions, by regulation of the enzymes involved in their biosynthesis 